\title{
Cryoballoon atrial fibrillation ablation: Single-center safety and efficacy data using a novel cryoballoon technology compared to a historical balloon platform
}

\author{
Adriano N. Kochi MD, MSc ${ }^{1,2}$ (1) | Massimo Moltrasio MD ${ }^{1}$ | \\ Fabrizio Tundo MD, $\mathrm{PhD}^{1}$ ｜ Stefania Riva $\mathrm{MD}^{1}$ | Ciro Ascione $\mathrm{MD}^{1}$ | \\ Maria A. Dessanai MD ${ }^{1}$ | Francesca Pizzamiglio MD $^{1}$ | Giulia Vettor MD ${ }^{1}$ \\ Selene Cellucci CCP ${ }^{1}$ | Alessio Gasperetti MD ${ }^{1}$ (1) | Claudio Tondo MD, PhD ${ }^{1,3}$ \\ Gaetano Fassini MD ${ }^{1}$
}

${ }^{1}$ Heart Rhythm Center at Centro Cardiologico Monzino IRCCS, Milan, Italy

${ }^{2}$ Nossa Senhora da Conceição Hospital, Porto Alegre, Brazil

${ }^{3}$ Department of Biochemical, Surgical and Dentist Sciences, University of Milan, Milan, Italy

\section{Correspondence}

Adriano N. Kochi, Heart Rhythm Center Centro Cardiologico Monzino IRCCS, Via

Carlo Parea, 4. Milan 20138, Italy.

Email: adrianokochi@gmail.com

\begin{abstract}
Introduction: Catheter ablation is superior to drugs regarding atrial fibrillation (AF) recurrence, symptoms improvement, and mortality reduction in heart failure. $\mathrm{POLARx}^{\mathrm{TM}}$ is a novel cryoballoon, with technical improvements seeking to improve outcomes. So far, its clinical evidence is restricted to a case report.

Methods: To compare the POLARx ${ }^{\mathrm{TM}}$ cryoballoon procedural safety and efficacy to the already established Arctic Front Advance $\mathrm{PRO}^{\mathrm{TM}}$ (AFAP) in a single-center cohort study, consecutive patients undergoing AF cryoablation with the POLARx ${ }^{\mathrm{TM}}$ were enrolled. Data were prospectively gathered. POLARx ${ }^{\mathrm{TM}}$ patients were compared with a historical cohort of patients submitted to AF cryoablation with the AFAP.

Results: Seventy patients were analyzed, 20 in POLARx ${ }^{\mathrm{TM}}$, and 50 in the AFAP group. They all underwent first-time pulmonary vein isolation, $77 \%$ were male, $94 \%$ had paroxysmal $\mathrm{AF}$, median age was 62.5 years, median $\mathrm{CHA}_{2} \mathrm{DS}_{2}-\mathrm{VASc} 1$, left-atrium size $34 \mathrm{ml} / \mathrm{m}^{2}$, and $65 \%$ were receiving anticoagulation. The primary end-point, all pulmonary veins isolation, was $100 \%$ in both groups. The complication rate was similar $10 \%$ POLARx $^{\mathrm{TM}}$ vs. 5.7\% AFAP, $p=.39$ ). The median total procedural time was longer in the POLARx ${ }^{\mathrm{TM}}$ group (90 min vs. $60 \mathrm{~min}, p<.001$ ), but the overall time-to-isolation (TTI; $44.8 \mathrm{~s}$ vs. $39 \mathrm{~s}, p=.253$ ) and ablation time (15 min vs. $13.7 \mathrm{~min}, p=.122$ ) was similar between POLARx ${ }^{\mathrm{T} M}$ and AFAP groups, respectively. Despite equal TTI, the POLARx ${ }^{\mathrm{TM}}$ had a lower minimal temperature reached $\left(-57^{\circ} \mathrm{C}\right.$ vs $\left.-47^{\circ} \mathrm{C}, p<.001\right)$.

Conclusion: The novel POLARx ${ }^{\mathrm{TM}}$ cryoballoon had similar efficacy and safety compared with the AFAP. It was also associated with longer procedural times, similar TTI, and lower minimum temperature reached.
\end{abstract}

\footnotetext{
Abbreviations: AFAP, Arctic Front Advance PRO; CB, cryoballoon; CKD, chronic kidney disease; CTI, cavotricuspid isthmus; DMS, diaphragmatic movement sensor; DOAC, direct oral anticoagulant; hs-Tnl, high-sensitive troponin I; LA, left atrium; LAAO, left atrial appendage occlusion; LIPV, left inferior pulmonary vein; LSPV, left superior pulmonary vein; LVEF, left ventricle ejection fraction; Min, minutes; N, number of patients; PVI, pulmonary vein isolation; PW, posterior wall; RF, radiofrequency; RIPV, right inferior pulmonary vein; RSPV, right superior pulmonary vein; Sec, seconds; SVC, superior vena cava; TIA, transient ischemic attack; VKA, vitamin $\mathrm{K}$ antagonist.
} 


\section{KEYWORDS}

ablation, Arctic Front Advance PRO, arrhythmias, atrial fibrillation, cryoablation, cryoballoon, POLARX

\section{$1 \mid$ INTRODUCTION}

Atrial fibrillation (AF) is the most common sustained arrhythmia worldwide. According to the Global Burden of Disease study, it affects 33.5 million individuals and its incidence is increasing every decade. ${ }^{1}$ Consequences are cerebrovascular events, heart failure, impaired quality of life, and increased mortality. ${ }^{2,3}$ Multiple trials have shown that catheter ablation is superior to antiarrhythmic drugs in reducing AF recurrence, alleviating symptoms, and mortality, in heart failure context. ${ }^{4-6}$

Cryoablation is a valid option to the classical point-by-point radiofrequency (RF) pulmonary vein isolation (PVI), with well-designed, randomized studies showing noninferiority when compared with RF catheter ablation. ${ }^{7,8}$ The Medtronic's cryoballoon (CB) overtime passed through many modifications seeking to improve quality. Compared with the first-generation CB Arctic Front ${ }^{\mathrm{TM}}$ (CB-1; Medtronic), Arctic Front Advance $^{\mathrm{TM}}$ (CB-2; Medtronic) presented enhanced distal hemisphere freezing, leading to a higher rate of acute PVI, shorter procedural times, and comparable safety. ${ }^{9}$ The Arctic Front Advance ST ${ }^{\mathrm{TM}}$ (CB-3; Medtronic) had a shorter distal nose-tip on the balloon, which facilitated the real-timed assessment of pulmonary vein potentials. ${ }^{10}$ Currently, most AF cryoablations are performed using the Arctic Front Advance PRO ${ }^{\text {TM }}$ system (Medtronic), the 4th generation device, characterized by better catheter maneuverability and ergonomics, achieving shorter procedural times. ${ }^{11}$

Recently, the POLARx ${ }^{\mathrm{TM}}$ (Boston Scientific) cryoballoon received CE-Mark and started to be used in Europe. The system has the same cryoablation workflow of the state-of-the-art cryoablation technology, with some improvements to, theoretically, improve outcomes. So far, its clinical evidence is restricted to a case report. ${ }^{12}$ Thus, we decided to perform this study comparing the POLARx ${ }^{\mathrm{TM}} \mathrm{CB}$ to the already established Arctic Front Advance PRO ${ }^{\mathrm{TM}}$.

\section{2 | METHODS}

\subsection{Study population}

The cohort study consisted in prospectively collecting data of the first 20 patients undergoing PVI for AF with the novel POLARx ${ }^{\mathrm{TM}}$ CB. They were compared to a historical cohort took from a study that evaluated the Arctic Front Advance PRO ${ }^{\mathrm{TM}}$ balloon (CB-4) against its previous version. ${ }^{13}$ The option for choosing this particular group of controls was
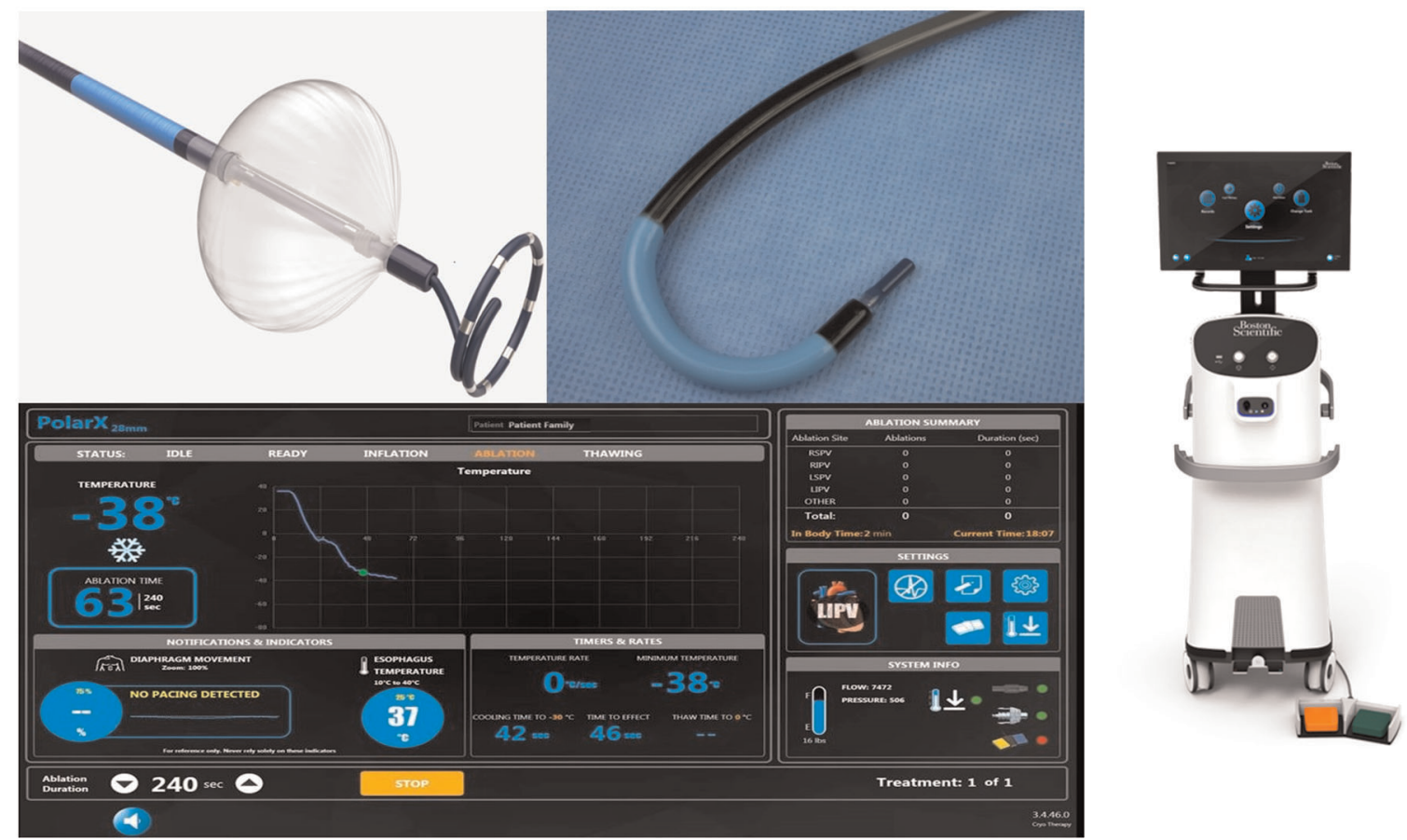

FIGURE 1 POLARX ${ }^{\mathrm{TM}}$ system. In the left upper panel: the POLARX ${ }^{\mathrm{TM}}$ cryoballoon and POLARMAP ${ }^{\mathrm{TM}}$ circular diagnostic catheter. In the center upper panel: the POLARSHEATH ${ }^{\mathrm{TM}}$ steerable sheath. In the lower panel: The SMARTFREEZE ${ }^{\mathrm{TM}}$ console screen. In the right side: the console and the pedal used to inflate/deflate the balloon and to initiate/stop cryoenergy delivery 
due to convenience and completeness of the clinical and procedural data available. In this case, the whole CB-4 group had data gathered for comparison. The project and data collection activities conform to the principles outlined in the Declaration of Helsinki, and each patient included provided informed written consent. The study was approved by the local Institutional Committee on Human Research.

\subsection{Aims of the study}

This study aims to evaluate the efficacy and safety of the POLARx ${ }^{\mathrm{TM}}$ $\mathrm{CB}$, comparing it with the established Arctic Front Advance PRO ${ }^{\mathrm{TM}}$. The chosen primary endpoint was acute success, defined as success in electrically isolating all the four veins in the procedure. Secondary outcomes were: complications rate, procedural duration, fluoroscopy time, ablation time, time-to-isolation (TTI), lowest temperature reached, number of veins isolated with a single cryo cycle, and highsensitive troponin I (hs-Tnl) level $24 \mathrm{~h}$ after the procedure.

\section{3 | Procedural workflow}

All the procedures were performed under deep sedation. A decapolar catheter was positioned in the coronary sinus and a quadripolar in superior vena cava (SVC). Intravenous unfractionated heparin was administered (100 UI/kg), aiming for an activated clotting time (ACT) between 350 and $450 \mathrm{~s}$. Standard fluoroscopy-guided transseptal puncture was performed, and the SLO sheath was changed over-the-wire for a Flexcath ${ }^{\mathrm{TM}} 15 \mathrm{Fr}$ or a POLARSHEATH ${ }^{\mathrm{TM}} 16 \mathrm{Fr}$. The veins were ablated following the sequence: left superior pulmonary vein (LSPV), left inferior pulmonary vein (LIPV), right inferior pulmonary vein (RIPV), and right superior pulmonary vein (RSPV). The vein occlusion was checked with contrast dye injection. The freezing time duration and the number of attempts for each vein, as well as additional lesions or procedures, were left to the operator's discretion. Usually, applications would vary from 180 to $300 \mathrm{~s}$, without bonus freeze. During ablation, vein potentials were monitored in real-time whenever possible by a circular wire-catheter (Achieve $^{\mathrm{TM}}$ or POLARMAP' ${ }^{\mathrm{TM}}$ ). When right pulmonary veins were ablated, the right phrenic nerve was paced through the SVC catheter, and diaphragmatic movements were closely monitored by upperabdomen palpation or movement sensor in case of POLARX ${ }^{\mathrm{TM}}$, triggering immediate freezing stop in case of ceasing or weakening of diaphragmatic movements. Esophageal temperature was monitored throughout the procedure. An esophageal temperature of less than $18^{\circ} \mathrm{C}$ would trigger immediate freeze cessation. After ablation, entrance block was confirmed by the absence of vein potentials, and exit block by failure in capturing the left atrium by stimulating inside the vein with maximum output. The POLARx ${ }^{\mathrm{TM}}$ console screen and gears are displayed in Figure 1. To see in detail the freezing-thawing process using the POLARx ${ }^{\mathrm{TM}}$ system, together with the built-in diaphragm movement sensor, watch the Video S1. Twenty-four hours after ablation all patients had hs-Tnl levels measured.

\section{4 | Statistical analysis}

Continuous variables were evaluated for normality using the Shapiro-Wilk test. Due to their non-normal distribution, they were described as median (Q1-Q3). Mann-Whitney U test was used for comparisons between them. Categorical variables were expressed as number (\%) and were analyzed using the $\chi^{2}$ or Fisher's exact test. Since this is an exploratory study, with no equal previous study, no sample size calculation was performed. Statistical tests were based on a two-sided significance level of 0.05. SPSS statistical software v.23.0 (IBM) was used for all statistical analyzes.

TABLE 1 Baseline clinical characteristics

\begin{tabular}{|c|c|c|c|c|}
\hline $\begin{array}{l}\text { Clinical } \\
\text { characteristics }\end{array}$ & $\begin{array}{l}\text { All patients } \\
(n=70)\end{array}$ & $\begin{array}{l}\text { POLARx } \\
(n=20)\end{array}$ & $\begin{array}{l}\text { Arctic } \\
\text { Front PRO } \\
(n=50)\end{array}$ & $p$ value \\
\hline Male gender & $54(77.1)$ & $12(60)$ & $42(84)$ & .056 \\
\hline Age & $62.5(55-69)$ & $63(59.6)$ & $61(53-70.2)$ & .984 \\
\hline Paroxysmal & $66(94.3)$ & $19(95)$ & $47(94)$ & 1.00 \\
\hline Hypertension & $27(38.6)$ & $12(60)$ & $15(30)$ & .03 \\
\hline Diabetes & $4(5.7)$ & $1(5)$ & $3(6)$ & 1.00 \\
\hline $\begin{array}{l}\text { Previous } \\
\text { stroke/TIA }\end{array}$ & $5(7.1)$ & $1(5)$ & $4(8)$ & 1.00 \\
\hline CKD & $1(1.4)$ & $0(0)$ & $1(2)$ & 1.00 \\
\hline Smoker & 12 (17.1) & $2(10)$ & $10(20)$ & .487 \\
\hline CAD & $10(14.3)$ & $1(5)$ & 9 (18) & .262 \\
\hline $\mathrm{CHA}_{2} \mathrm{DS}_{2}$-VASc & $1(0-2.25)$ & $1.5(0-2.0)$ & $1.0(0-3.0)$ & .606 \\
\hline Betablocker & $28(40)$ & $10(50)$ & $18(36)$ & .295 \\
\hline $\begin{array}{l}\text { Calcium } \\
\text { antagonist }\end{array}$ & $9(12.9)$ & $2(10)$ & $7(14)$ & 1.00 \\
\hline ACE-inhibitor & $28(40)$ & $10(50)$ & $18(36)$ & .295 \\
\hline Antiagregation & $6(8.6)$ & $2(10)$ & $4(8)$ & 1.00 \\
\hline $\begin{array}{l}\text { Antiarrhythmic } \\
\text { drugs }\end{array}$ & & & & .228 \\
\hline $\begin{array}{l}\text { Sodium } \\
\text { blockers }\end{array}$ & $34(48.5)$ & $10(50)$ & $23(46)$ & \\
\hline Sotalol & $2(2.8)$ & $2(1)$ & $0(0)$ & \\
\hline Amiodarone & $14(20)$ & $3(15)$ & $11(22)$ & \\
\hline Anticoagulants & & & & .816 \\
\hline DOAC & $45(64.3)$ & $13(65)$ & $32(64)$ & \\
\hline VKA & $1(1.4)$ & $0(0)$ & $1(2)$ & \\
\hline $\begin{array}{l}\text { LA size } \\
\qquad\left(\mathrm{ml} / \mathrm{m}^{2}\right)\end{array}$ & $34(31-42)$ & $36(30-41)$ & $33(31-43)$ & .970 \\
\hline LVEF (\%) & $62(57-67)$ & $60(57-66)$ & $62(56.7-67)$ & $.412::$ \\
\hline
\end{tabular}

Note: Variables are presented as number (\%) or median (Q1-Q3).

Abbreviations: ACE, angiotensin-converting enzyme; $C A D$, coronary artery disease; CKD, chronic kidney disease; DOAC, direct oral anticoagulants; LA, left atrium; LVEF, left ventricular ejection fraction; TIA, transient ischemic attack; VKA, vitamin $\mathrm{K}$ antagonist. 


\section{3 | RESULTS}

A total of 70 patients were analyzed in this study, 20 from the POLARx ${ }^{T M}$ group, enrolled from August 12, 2020 to October 30, 2020; and 50 from the Arctic Front Advance PRO ${ }^{\mathrm{TM}}$, sampled from a cohort of a previously published study (from October 2018 to February
2019). Their median age was 62.5 years, the majority were male (77.1\%), had paroxysmal AF (94.3\%), a median LA size of $32 \mathrm{ml} / \mathrm{m}^{2}$, and $\mathrm{CHA}_{2} \mathrm{DS}_{2}$-VASc 1. Except for hypertension, the baseline clinical characteristics were similar between groups. All patients were undergoing their first PVI procedure. Only three patients had common trunk, all in the left, one in the POLARx group and the other two in the

\begin{tabular}{|c|c|c|c|c|}
\hline Variable & All $(n=70)$ & POLARx $(n=20)$ & $\begin{array}{l}\text { Arctic Front } \\
\text { Advance } \\
\text { PRO }(n=50)\end{array}$ & $p$ value \\
\hline Duration (min) & $60(50-80)$ & $90(60-120)$ & $60(50-72)$ & $<.001$ \\
\hline Ablation time (min) & 15 (13.3-16) & $15(13.5-20.7)$ & $15(13-16)$ & .122 \\
\hline Fluoroscopy (min) & $13.9(10.8-18.2)$ & 15 (11.9-21.2) & $13.7(10.6-17.7)$ & .291 \\
\hline \multicolumn{5}{|l|}{ TTI (s) } \\
\hline Overall & $39.6(29.1-56.1)$ & $44.8(28-64.4)$ & $39(29-51.6)$ & .253 \\
\hline LSPV & $40(30-60)$ & $44(29-66)$ & $39(30-52.5)$ & .255 \\
\hline LIPV & $34.5(22-63.5)$ & $35.5(24-63.5)$ & $33.5(21.2-67.5)$ & .449 \\
\hline RSPV & $29(22-47)$ & $32(23-50)$ & $29(18.5-44.2)$ & .361 \\
\hline RIPV & $30(25-49.5)$ & $31(25.7-80)$ & $30(20.7-48.5)$ & .612 \\
\hline
\end{tabular}

TABLE 2 Procedural outcomes

Temperature TTI $\left({ }^{\circ} \mathrm{C}\right)$

$\begin{array}{lllll}\text { Overall } & -34(-29.5 ;-40.5) & -44(-36 ;-50) & -32(-28.3 ;-36.6) & <.001 \\ \text { LSPV } & -36(-33 ;-45) & -52(-43.5 ;-53.2) & -35(-33 ;-41) & <.001 \\ \text { LIPV } & -35(-24.7 ;-41.5) & -47(-36 ;-50) & -32(-21.5 ;-39) & .001 \\ \text { RSPV } & -34(-23.7 ;-41) & -40(-20 ;-49.5) & -33(-23.2 ;-38) & .246 \\ \text { RIPV } & -34(-28 ;-41) & -42(-34.5 ;-50) & -32(-27.5 ;-38) & .001\end{array}$

Lowest temperature $\left({ }^{\circ} \mathrm{C}\right)$

$\begin{array}{lllll}\text { Overall } & -49.3(-45.8 ;-55.5) & -57(-55.6 ;-60.2) & -47.5(-44.9 ;-50) & <.001 \\ \text { LSPV } & -52(-46 ;-58) & -60(-58 ;-60) & -49(-44.2 ;-52.7) & <.001 \\ \text { LIPV } & -48(-44 ;-53.7) & -56(-52 ;-61) & -46(-43 ;-50) & <.001 \\ \text { RSPV } & -51(-47 ;-57,2) & -59(-55.2 ;-64) & -50(-46 ;-52.2) & <.001 \\ \text { RIPV } & -49(-44 ;-56) & -57.5(-52 ; 63.7) & -47(-42.5 ;-51) & <.001 \\ \begin{array}{l}\text { Veins isolated in the } \\ \text { first attempt }\end{array} & 4(3-4) & 3(2.0-3.75) & 4(3.5-4.0) & <.001 \\ \end{array}$

\begin{tabular}{lllll} 
Combined procedure & & & \\
Overall & $19(27.1)$ & $3(15)$ & $16(32)$ & .234 \\
CTI line & $16(22.9)$ & $1(5)$ & $15(30)$ & .028 \\
LAAO & $2(2.9)$ & $1(5)$ & $1(2)$ & .493 \\
PW isolation & $1(1.4)$ & $1(5)$ & 0 & .822 \\
hs-Tnl 24h after & $8591(5916-11,197)$ & $9006(6020-11,073)$ & $8369(5810-11,511)$ & .39 \\
\hline Complications & $4(5.7)$ & $0(0)$ & $4(8)$ & 1.00 \\
\hline Acute success & $70(100)$ & $20(100)$ & $50(100)$ & \\
\hline
\end{tabular}

Note: Variables are displayed as median (Q1-Q3) or median (Q1;Q3).

Abbreviations: CTI, cavotricuspid isthmus; hs-Tnl, high-sensitive troponin I; LAAO, left atrial appendage occlusion; LIPV, left inferior pulmonary vein; LSPV, left superior pulmonary vein; Min, minutes; PW, posterior wall; RIPV, right inferior pulmonary vein; RSPV, right superior pulmonary vein; $\mathrm{s}$, seconds; TTI, time-to-isolation. 

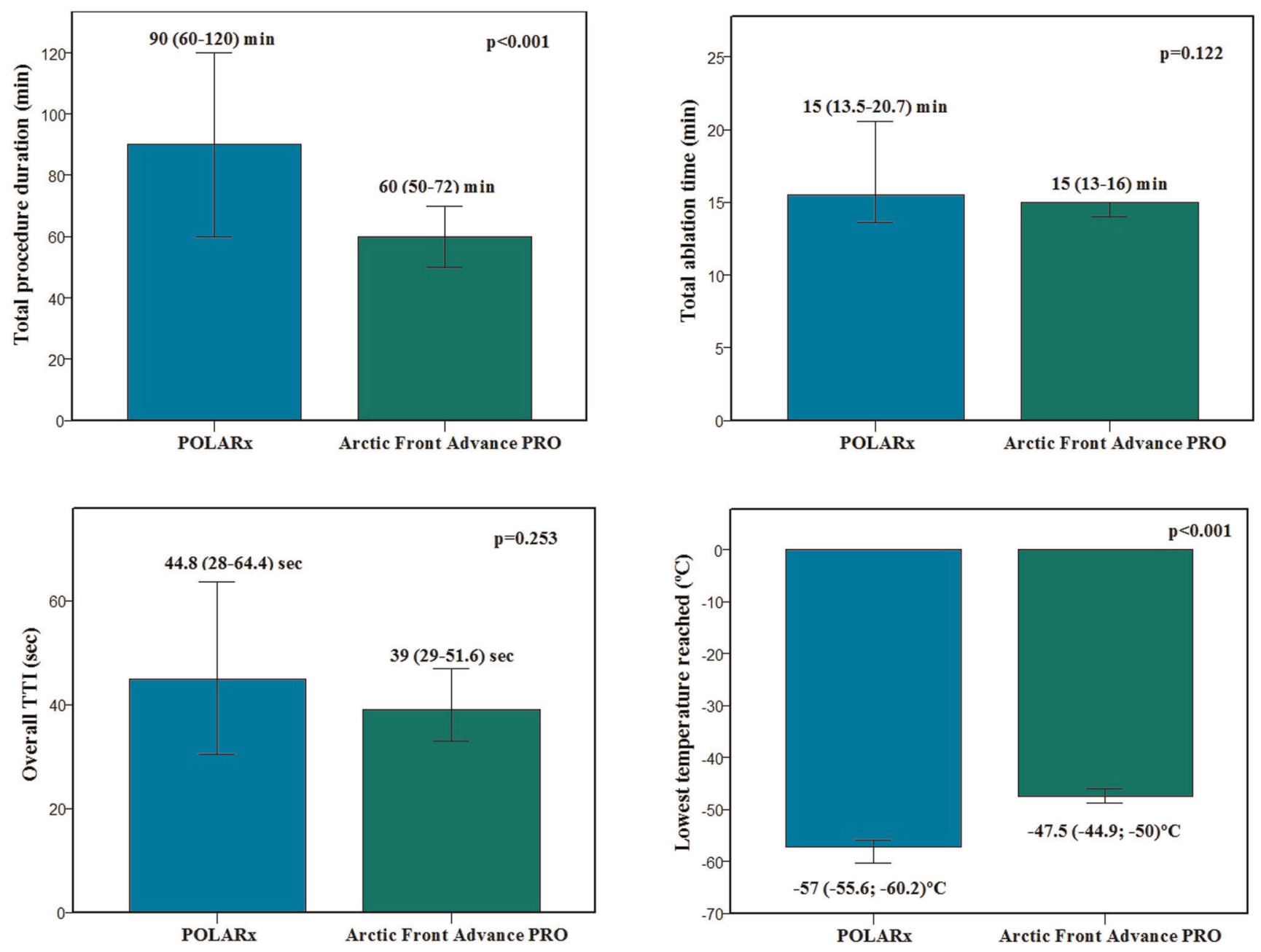

FIGURE 2 Boxplots showing procedural data

Arctic Front Advance PRO group. Due to the small number, they were not considered for statistical analysis of data regarding left veins ablation. Details about baseline clinical characteristics can be seen in Table 1. The primary efficacy endpoint, acute success, was obtained in $100 \%$ of patients in both groups. Complications occurred exclusively in the Arctic Front Advance PRO ${ }^{\mathrm{TM}}$ group, but in a small number (0 vs. 4 [8\%], $p=.39$ ), among them, three self-limited phrenic palsies and a postprocedural pericardial effusion without necessity for intervention. Compared with Medtronic's CB, POLARx ${ }^{\mathrm{TM}}$, in this cohort, was associated with a longer procedure $(90 \mathrm{~min}$ vs. $60 \mathrm{~min}, p<.001$ ), lower minimum temperature $\left(-57^{\circ} \mathrm{C}\right.$ vs. $\left.-47.5^{\circ} \mathrm{C}, p<.001\right)$, lower TTI temperature $\left(-44^{\circ} \mathrm{C}\right.$ vs. $\left.-32^{\circ} \mathrm{C}, p<.001\right)$, but similar TTI $(44.8 \mathrm{~s}$ vs. $39 \mathrm{~s}$, $p<.001)$. The median number of veins isolated in the first attempt, per patient, was also lower in POLARx ${ }^{\mathrm{TM}}$ CB ( 3 vs. $4, \mathrm{p}<0.001$ ). No patients had necessity to undergo touch-up ablation with RF to complete vein isolation. Overall $27.1 \%$ of patients underwent a combined procedure, mostly cavotricuspid ablation line for typical atrial flutter $(84.2 \%$ of the combined procedures). No difference in the hs-Tnl level collected $24 \mathrm{~h}$ after the procedure was observed between groups. The full procedural details are displayed in Table 2. They are also displayed as plot in Figure 2.

\section{4 | DISCUSSION}

To the best of your knowledge, this is the first clinical study comparing the novel POLARx ${ }^{\mathrm{TM}} \mathrm{CB}$ to the already established Arctic Front Advance PRO ${ }^{\mathrm{TM}}$.

Briefly, we found that the POLARx ${ }^{\mathrm{TM}} \mathrm{CB}$ had a similar profile in terms of efficacy and safety when compared with the Medtronic's CB. Despite achieving all veins isolation by the end of the procedure in $100 \%$ of the patients, the POLARx ${ }^{\mathrm{TM}}$ CB demanded a higher procedural time and had a lower number of veins isolated in the first attempt. Interestingly, the cryoenergy application time was the same. A possible explanation for that could be the lack of experience with some of the new features and workflow of the POLARx ${ }^{\mathrm{TM}}$, an issue intrinsic to the first procedures with new devices. Notwithstanding the similarities between tools and procedural workflow, some differences in the practical handling were consensus between our operators. The POLARSHEATH ${ }^{\mathrm{T} M}$ introducer, although $1 \mathrm{Fr}$ larger, tended to cross the septum more easily and smoothly due to its more gradual taper from the dilator to the sheath. We also had the feeling that the set sheath plus balloon was softer and more flexible when compared to the Flexcath $^{\mathrm{TM}}$ plus $\mathrm{CB}$. This can be seen as an advantage since it allows 


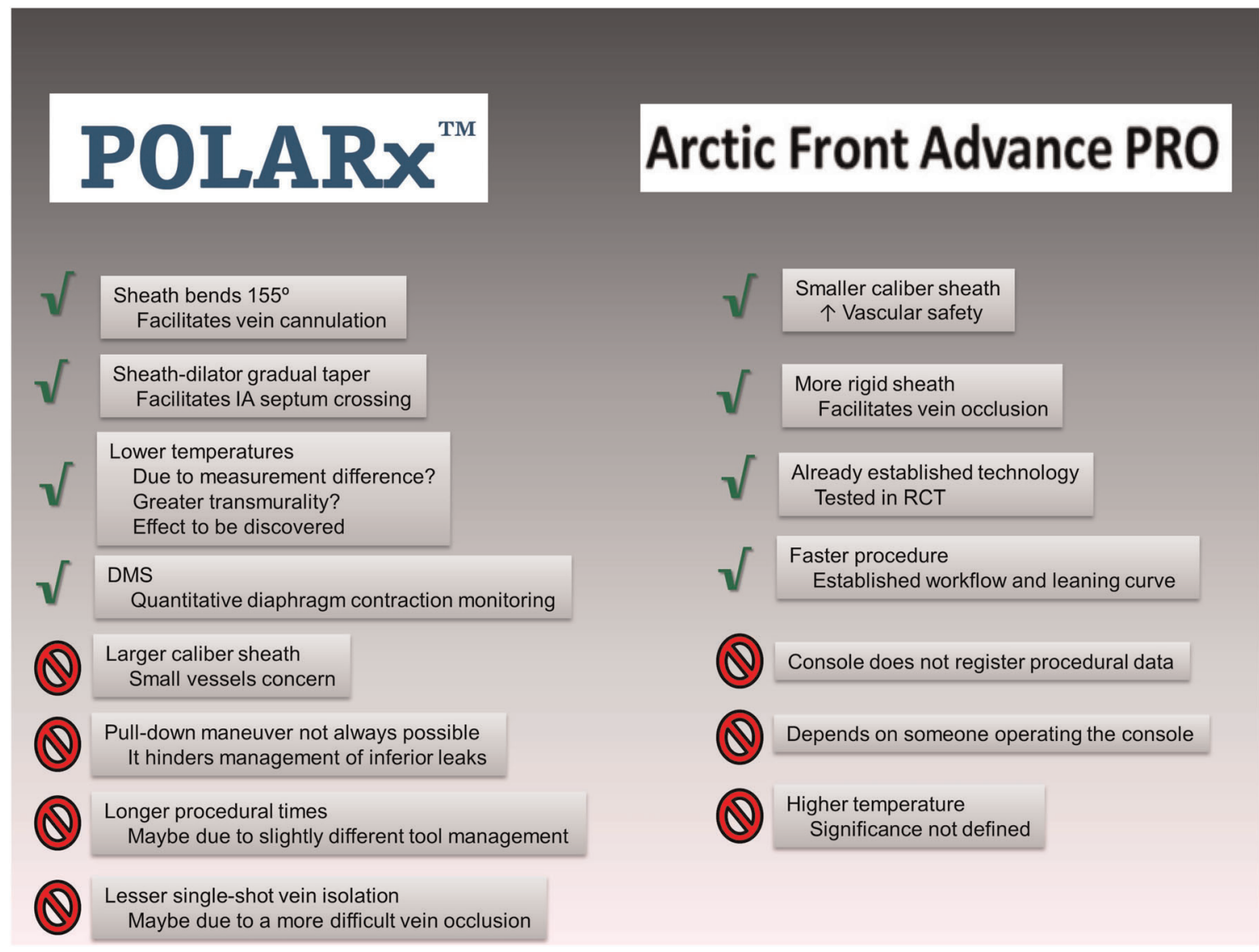

FIGURE 3 Pros and cons of both technologies

easier vein cannulation, or disadvantage once it can make more difficult to reach vein occlusion. Another point against the novel $C B$ is the impossibility to perform the pull-down maneuver. This is due to a build-in sensor of balloon dislodgement aiming to enhance procedural safety. When the CB is pulled to seal the inferior vein segment, the system often detects it as an involuntary CB displacement and it stops the cryoenergy delivery immediately, hindering a maneuver commonly used to seal the vein.

An interesting finding is the difference found in the minimum temperature reached. The median lowest temperature and temperature reached when the vein turned isolated was approximately $10^{\circ} \mathrm{C}$ lower in the POLARx ${ }^{\mathrm{TM}}$ group. Despite that, the TTI was similar between groups, and so does the troponin level. These findings point to a non-different tissue damage, making us theorize that the temperature inside the atrial tissue does not differ between groups, and that this temperature difference may be due to a different way or location where it is measured. Since we did not perform routinely pre or post voltage map, we were not able to evaluate acute and/or chronic scar extension. In one of the POLARx ${ }^{\mathrm{TM}}$ patients, we performed successfully, besides PVI, posterior wall isolation exclusively using the $\mathrm{CB}$; the result can be seen in a previous publication. ${ }^{12}$

No complication was observed in the 20 POLARx $^{\mathrm{TM}}$ cases, and four minor complications were observed in the Arctic Advance PRO ${ }^{\mathrm{TM}}$ group: three temporary phrenic nerve palsies and a pericardial effusion without hemodynamic compromise. Since, fortunately, as in other AF cryoablation cohorts, our complication incidence was low, we could not draw significant conclusions about group differences. Another interesting feature of POLARx ${ }^{\mathrm{TM}}$ is the possibility to monitor diaphragm contraction with the diaphragmatic movement sensor (DMS), a sensor that shows it in real-time as a quantified number in percentage. If even a reduction in diaphragm contraction is detected it triggers a red warning sign, allowing earlier cryoenergy delivery stop. Unfortunately, it is not always possible to use the DMS together with hand palpation, since the pressure applied by the hand cause error in the DMS reading, as can be seen in Video S1. The summary of pros and cons of both technologies is available in Figure 3.

Owing to the fact that POLARx ${ }^{\mathrm{TM}}$ has just entered the market in Europe, no data from previous studies is available to be compared with ours. But, taking into account previous studies comparing different $C B$ generations, we observe that evolution is possible and desired. Since the release of the first generation (CB-1), Medtronic improved the design three times, being born: Arctic Front Advance ${ }^{\mathrm{TM}}$ (CB-2), Arctic Front Advance ST ${ }^{\mathrm{TM}}$ (CB-3), and lastly, the Arctic Front Advance $\mathrm{PRO}^{\mathrm{TM}}$ (CB-4). A real-world study evaluating 480 patients, 120 in each generation group, found a progressive reduction in procedural duration (150 min, $95 \mathrm{~min}, 90 \mathrm{~min}$, and $75 \mathrm{~min}$, from CB-1 to $\mathrm{CB}-4$, as well as a reduction in fluoroscopy time and ablation duration, with the same success rate. This denotes that even if 
reaching a higher success was not possible, the evolution in balloon architecture improved the handling, making the procedure easier. ${ }^{11}$

Summarizing, our study found that the novel POLARx ${ }^{\text {TM }}$ CB had similar efficacy and safety compared with the Arctic Front Advance $\mathrm{PRO}^{\text {TM }} \mathrm{CB}$. It also achieved lower temperatures, but with similar TTIs. However, it was associated with a longer procedure and a lower number of pulmonary veins isolated in the first attempt. The DMS for phrenic monitoring is useful and has the potential to make the procedure safer.

\section{5 | LIMITATIONS}

Among the limitation are its non-randomized study design, the small sample size since the new balloon has just entered the market, and the impossibility to blind the operator once each catheter has marked physical differences. In this study, a follow-up study was not possible because we performed the last cases a few weeks before writing the manuscript.

\section{6 | CONCLUSION}

The novel POLARx ${ }^{\mathrm{TM}}$ cryoballoon had similar efficacy and safety when compared with the Arctic Front Advance Balloon ${ }^{\mathrm{TM}}$. Since this is the first study to evaluate it, future studies with larger sample sizes are necessary to confirm our findings, especially about a possible reduction in complications.

\section{ACKNOWLEDGMENT}

This study did not receive any specific grant from funding agencies in the public, commercial, or not-for-profit sectors.

\section{CONFLICT OF INTERESTS}

The authors declare that there are no conflict of interests.

\section{PATIENT CONSENT STATEMENT}

The project and data collection activities conform to the principles outlined in the Declaration of Helsinki, and each patient included provided informed written consent.

\section{DATA AVAILABILITY STATEMENT}

The data that support the findings of this study are available from the corresponding author upon reasonable request.

\section{ETHICS STATEMENT}

The study was approved by the local Institutional Committee on Human Research.

\section{ORCID}

Adriano N. Kochi (D) https://orcid.org/0000-0002-7839-2054 Alessio Gasperetti (D) http://orcid.org/0000-0003-3432-070X

\section{REFERENCES}

1. Chugh SS, Havmoeller R, Narayanan K, et al. Worldwide epidemiology of atrial fibrillation: A global burden of disease 2010 study. Circulation. 2014;129:837-847.

2. Zoni-Berisso M, Lercari F, Carazza T, Domenicucci S. Epidemiology of atrial fibrillation: European perspective. Clin Epidemiol. 2014;6:213-220.

3. Hindricks G, Potpara T, Dagres N, et al. ESC Guidelines for the diagnosis and management of atrial fibrillation developed in collaboration with the European Association of Cardio-Thoracic Surgery (EACTS). Eur Heart J. 2020;2020:1-126.

4. Packer DL, Kowal RC, Wheelan KR, et al. Cryoballoon ablation of pulmonary veins for paroxysmal atrial fibrillation: first results of the North American arctic front (STOP AF) pivotal trial. J Am Coll Cardiol. 2013;61:1713-1723.

5. Packer DL, Mark DB, Robb RA, et al. Effect of catheter ablation vs antiarrhythmic drug therapy on mortality, stroke, bleeding, and cardiac arrest among patients with atrial fibrillation: the CABANA randomized clinical trial. JAMA - J Am Med Assoc. 2019;321:1261-1274.

6. Cosedis Nielsen J, Johannessen A, Raatikainen P, et al. Radiofrequency ablation as initial therapy in paroxysmal atrial fibrillation. N Engl J Med. 2012;367:1587-1595.

7. Kuck $\mathrm{KH}$, Brugada J, Fürnkranz $\mathrm{A}$, et al. Cryoballoon or radiofrequency ablation for paroxysmal atrial fibrillation. $\mathrm{N} \mathrm{Engl} \mathrm{J} \mathrm{Med.}$ 2016;374:2235-2245

8. Andrade JG, Deyell MW, Verma A, MacLe L, Khairy P. The cryoballoon vs irrigated radiofrequency catheter ablation (CIRCA-DOSE) study results in context. Arrhythmia Electrophysiol Rev. 2020;9:34-39.

9. Coulombe N, Paulin J, Su W. Improved in vivo performance of second-generation cryoballoon for pulmonary vein isolation. J Cardiovasc Electrophysiol. 2013;24:919-925.

10. Aryana A, Kowalski M, O'Neill PG, et al. Catheter ablation using the third-generation cryoballoon provides an enhanced ability to assess time to pulmonary vein isolation facilitating the ablation strategy: Short- and long-term results of a multicenter study. Hear Rhythm. 2016;13:2306-2313.

11. lacopino S, Pieragnoli P, Arena G, et al. A comparison of acute procedural outcomes within four generations of cryoballoon catheters utilized in the real-world multicenter experience of 1STOP. J Cardiovasc Electrophysiol. 2020;31:80-88.

12. Moltrasio M, Kochi AN, Fassini G, Riva S, Tundo F, Tondo C. Highdensity mapping validation of antral pulmonary vein isolation and posterior wall isolation created with a new cryoballoon ablation system: The first reported case. J Cardiovasc Electrophysiol. 2020;31: 3318-3321. https://doi.org/10.1111/jce.14741

13. Moltrasio $M$, Sicuso R, Fassini GM, et al. Acute outcome after a single cryoballoon ablation: comparison between Arctic Front Advance and Arctic Front Advance PRO. PACE - Pacing Clin Electrophysiol. 2019;42:890-896.

\section{SUPPORTING INFORMATION}

Additional Supporting Information may be found online in the supporting information tab for this article.

How to cite this article: Kochi AN, Moltrasio M, Tundo F, et al. Cryoballoon atrial fibrillation ablation: Single-center safety and efficacy data using a novel cryoballoon technology compared to a historical balloon platform. J Cardiovasc Electrophysiol. 2021;1-7. https://doi.org/10.1111/jce.14930 